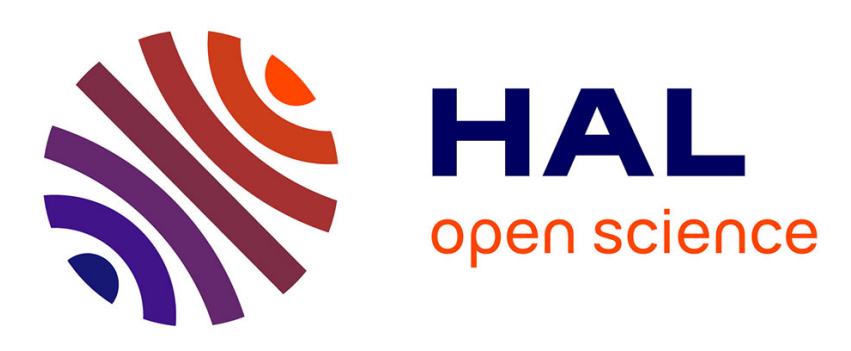

\title{
Game-Theoretic Centrality Measures for Weighted Graphs
}

Vladimir Mazalov, Konstantin Avrachenkov, Liudmila Trukhina, Bulat

Tsynguev

\section{- To cite this version:}

Vladimir Mazalov, Konstantin Avrachenkov, Liudmila Trukhina, Bulat Tsynguev. Game-Theoretic Centrality Measures for Weighted Graphs. Fundamenta Informaticae, 2016, Discrete Mathematics (RuFiDiM 14), 145 (3), pp.341-358. 10.3233/FI-2016-1364 . hal-01402858

\section{HAL Id: hal-01402858 \\ https://hal.inria.fr/hal-01402858}

Submitted on 25 Nov 2016

HAL is a multi-disciplinary open access archive for the deposit and dissemination of scientific research documents, whether they are published or not. The documents may come from teaching and research institutions in France or abroad, or from public or private research centers.
L'archive ouverte pluridisciplinaire HAL, est destinée au dépôt et à la diffusion de documents scientifiques de niveau recherche, publiés ou non, émanant des établissements d'enseignement et de recherche français ou étrangers, des laboratoires publics ou privés. 
DOI 10.3233/FI-2012-0000

IOS Press

\title{
Game-Theoretic Centrality Measures for Weighted Graphs
}

\author{
Mazalov V.V. \\ Institute of Applied Mathematical Research \\ of the Karelian Research Center RAS \\ Petrozavodsk, Russia \\ vlmazalov@yandex.ru

\section{Trukhina L.I.} \\ Chita Institute Baikal State University \\ of Economics and Law \\ Chita, Russia
}

\author{
Avrachenkov K.E. \\ Inria Sophia Antipolis \\ France \\ Tsynguev B.T. \\ Transbaikal State University \\ Chita, Russia
}

\begin{abstract}
The betweenness centrality is one of the basic concepts in the analysis of the social networks. Initial definition for the betweenness of a node in the graph is based on the fraction of the number of geodesics (shortest paths) between any two nodes that given node lies on, to the total number of the shortest paths connecting these nodes. This method has polynomial complexity. We propose a new concept of the betweenness centrality for weighted graphs using the methods of cooperative game theory. The characteristic function is determined by special way for different coalitions (subsets of the graph). Two approaches are used to determine the characteristic function. In the first approach the characteristic function is determined via the number of direct and indirect weighted connecting paths in the coalition. In the second approach the coalition is considered as an electric network and the characteristic function is determined as a total current in this network. We use the Kirchhoff's law. After that the betweenness centrality is determined as the Myerson value. The results of computer simulations for some examples of networks, in particular, for the popular social network "VKontakte", as well as the comparing with the PageRank method are presented.
\end{abstract}

Keywords: betweenness centrality, weighted graph, cooperative game, social networks. 


\section{Introduction}

The social networks give impulse to the development of new graph-theoretical methods for the analysis of networks. Social network analysis methods are applied in many fields: economics, physics, sociology, biology and information technologies.

One of the basic concepts in the analysis of the social networks is betweenness centrality, a measure of centrality that is based on how well a node $i$ is situated in terms of the paths that it lies on [9]:

$$
c_{B}(i)=\frac{1}{n_{B}} \sum_{s, t \in V} \frac{\sigma_{s, t}(i)}{\sigma_{s, t}},
$$

where $\sigma_{s, t}$ is the total number of geodesics (shortest paths) between nodes $s$ and $t, \sigma_{s, t}(i)$ is the number of geodesics between $s$ and $t$ that $i$ lies on. The denominator $n_{B}$ captures that the node $i$ could lie on paths between as many as $n_{B}=(n-1)(n-2) / 2$ pairs of other nodes. The complexity of the fastest algorithm to find $c_{B}(i)$ is $O(m n)$ and presented in [6].

To determine a variation of betweenness centrality we will use the methods of cooperative game theory using the model of the communication game developed by Myerson. Cooperative game is determined by the set of players $N=\{1,2, \ldots, n\}$ and a characteristic function $v(K)$, utility of a coalition $K$. There are many real situations where there is a restriction in the coalition formation. Here we consider a cooperative game with restricted cooperation which is presented by an undirected communication graph first developed by Myerson [16]. The network relations are formally represented by graphs whose nodes are identified with the players and whose arcs capture the pairwise relations. The relation can be interpreted as an information transfer or resource distribution or transport connection. The nodes can be individuals or organizations or countries or web-pages.

It brings a communication (networking) game determined by a triple which consists of a finite set of players, a characteristic function and a graph of relations between players. A useful solution of the communication game is the Myerson value which is characterized by the component efficiency and pairwise stability.

Efficiency means that for each component of the graph the common payoff is equal to the value of the component. The stability means that deleting or adding of a connection between two players leads to equal deviations in payoff allocation for both players.

Jackson and Wolinsky [11] proposed the model of networking game in which the utility depends of the structure of the network. They apply the Myerson value to analyse the betweenness of the nodes in the network. Despite the fact that other allocation rules were proposed (see, for instance, $[4,5,8,10,19$, 20, 21]), the Myerson vector is widely used as an allocation rule in many cooperative games.

But computing the Myerson value is not an easy problem. In the paper [15] a sufficiently simple procedure was proposed to calculate the Myerson value for a cooperative game on the unweighted graph with special characteristic function.

In $[7,17]$ a centrality measure is defined inspired by a model of electrical circuits. The communication graph is considered as an electrical network where the vertices of the graph are the network nodes and the links have some conductivity (reciprocal to resistance). To find the current in the network, the Kirchhoff's law is used. At a node $s$ we put a unit current and a node $t$ is grounded. The centrality measure of a node $i$ is a mean value of the transmitted current over the node $i$ for all possible pairs $s$ and $t$. Note that in the definition of the electric measure of centrality definition all possible paths for the 
current are used, not only shortest paths.

The complexity of the best algorithm to find the electric centrality is $O(I(n-1)+m n \log n)$, where $I(n-1)$ is a complexity to find the inverse matrix of the size $n-1[7,17]$.

In [2] the authors consider a model of electric circuit where the conductivities of the links are constant and equal to $\alpha$. In contrast to [7] in the model [2] a new grounded node $n+1$ is introduced and each node of the network is connected with this node with the link of conductivity $1-\alpha$. This approach allows to decrease the complexity of the algorithm to find the centrality. And hence, there is a possibility to compute the centrality for large graphs. However this approach was proposed only for unweighted graphs.

In this paper we propose a new concept of betweenness centrality for weighted graphs using the methods of cooperative game theory. The characteristic function is determined by special way for different coalitions (subsets of the graph). Two approaches are used to determine the characteristic function. In the first approach the characteristic function is determined by the number of direct and indirect connecting paths in the coalition [11]. At that, the weighted graph is transformed into multigraph and then we use the method proposed in [15].

In the second approach the coalition is considered as an electric network and the characteristic function is determined as a total current in this network. The current is determined using the Kirchhoff's law. After that the betweenness centrality is determined as the Myerson value. The results of computer simulations for some examples of networks, in particular, for the popular social network "VKontakte", as well as the comparing with the PageRank method are presented. Our main conclusion that the electric centrality measure correlates well with the game-theoretic based centrality measures.

\section{Cooperative game and the Myerson value}

The betweenness centrality in a network can be determined using game-theoretic approach. Let the nodes of the network correspond to the set of players in a cooperative game.

Let $N=\{1,2, \ldots, n\}$ be the finite set of players. Denote $2^{N}$ the set of all subsets of $N$. A subset $S \in 2^{N}$ is referred to as a coalition in cooperative game.

Cooperative game of $n$ players is a pair $\langle N ; w\rangle$ where $N=\{1,2, \ldots, n\}$ is the set of players and $w: 2^{N} \rightarrow R$ is the map prescribing for a coalition $S \in 2^{N}$ some value $w(S)$ such that $w(\emptyset)=0$. This function $w(S)$ is the total utility that members of $S$ can jointly attain. Such function is called the characteristic function of cooperative game.

Undirected graph $g=(N, E)$ consists of the set of nodes $N$ and the set of links $E$. We denote the link as $i j$. The interpretation is that if $i j \in E$, then the nodes $i \in N$ and $j \in N$ are directly connected in graph $g$, while if $i j \notin E$, then nodes $i$ and $j$ are not directly connected.

Let $N\left(g^{\prime}\right)=\left\{i: \exists j\right.$ such that $\left.i j \in g^{\prime}\right\}$. For a graph $g$, a sequence of different nodes $\left\{i_{1}, i_{2}, \ldots, i_{k}\right\}, k \geq$ 2 , is a path connecting $i_{1}$ and $i_{k}$ if for all $h=1, \ldots, k-1, i_{h} i_{h+1} \in g$. The length of the path, $l$, is the number of links in the path, i.e. $l=k-1$. The length of the shortest path connecting $i$ and $j$ is the distance between $i$ and $j$. Graph $g$ on the set $N$ is connected graph if for any two nodes $i$ and $j$ there exists a path in $g$ connecting $i$ and $j$.

The coalition $S$ is connected if any two nodes in $S$ are connected by a path which consists of nodes from $S$. The graph $g^{\prime}$ is a component of $g$, if for all $i \in N\left(g^{\prime}\right)$ and $j \in N\left(g^{\prime}\right)$, there exists a path in $g^{\prime}$ connecting $i$ and $j$, and for any $i \in N\left(g^{\prime}\right)$ and $j \in N(g), i j \in g$ implies that $i j \in g^{\prime}$. Let $N \mid g$ is the set 
of all components in $g$.

Let $g-i j$ denote the graph obtained by deleting link $i j$ from the graph $g$ and $g+i j$ denote the graph obtained by adding link $i j$ to the graph $g$. Let $\langle N, v\rangle$ is a cooperative game with partial cooperation presented by graph $g$ and characteristic function $v$. An allocation rule $Y$ describes how the value associated with the network is distributed to the individual players. $Y_{i}(v, g)$ is the value to player $i$ from graph $g$ under the characteristic function $v$.

Myerson proposed in [16] the allocation rule

$$
Y(v, g)=\left(Y_{1}(v, g), \ldots, Y_{n}(v, g)\right),
$$

which is uniquely determined by the following axioms:

A1. If $S$ is a component of $g$ then the members of the coalition $S$ ought to allocate to themselves the total value $v(S)$ available to them, i.e $\forall S \in N \mid g$

$$
\sum_{i \in S} Y_{i}(v, g)=v(S)
$$

A2. $\forall g, \forall i j \in g$ both players $i$ and $j$ obtain equal payoffs after adding or deleting a link $i j$,

$$
Y_{i}(v, g)-Y_{i}(v, g-i j)=Y_{j}(v, g)-Y_{j}(v, g-i j) .
$$

Let us determine the characteristic function by the following way

$$
v_{g}(S)=\sum_{K \in S \mid g} v(K)
$$

Then the Myerson value can be calculated by formula

$$
Y_{i}(v, g)=\sum_{S \subset N \backslash\{i\}}\left(v_{g}(S \cup i)-v_{g}(S)\right) \frac{s !(n-s-1) !}{n !},
$$

where $s=|S|, \quad n=|N|$.

\section{Centrality measure for weighted network using Myerson value}

Consider a game where the graph $g$ is a tree which consists on $n$ nodes and characteristic function is determined by the scheme proposed by Jackson [12]: every direct connection gives to coalition $S$ the impact $r$, where $0 \leq r \leq 1$. Moreover, players obtain an impact from non-direct connections. Each path of length 2 gives to coalition $S$ the impact $r^{2}$, a path of length 3 gives to coalition the impact $r^{3}$, etc. So, for any coalition $S$ we obtain

$$
v(S)=a_{1} r+a_{2} r^{2}+\cdots+a_{k} r^{k}+\cdots+a_{L} r^{L}=\sum_{k=1}^{L} a_{k} r^{k},
$$

where $L$ is a maximal distance between two nodes in the coalition; $a_{k}$ is the number of paths of length $k$ in this coalition.

$$
v(i)=0, \forall i \in N \text {. }
$$


Let us define the allocation rule in this cooperative game in the following form

$$
Y_{i}(w, g)=\frac{\sigma_{1}(i)}{2} r+\frac{\sigma_{2}(i)}{3} r^{2}+\cdots+\frac{\sigma_{L}(i)}{L+1} r^{L}=\sum_{k=1}^{L} \frac{\sigma_{k}(i)}{k+1} r^{k},
$$

where $\sigma_{k}(i)$ is a number of the paths of the length $k$ which include $i$. In [15] it is proven that this allocation rule for unweighted graphs is the Myerson value.

The Myerson value for player $i$ depends on the number of paths that it lies on. Consequently, large Myerson value corresponds to large number of paths which follow over this node. Therefore, Myerson value can be used as a centrality definition for the nodes in the network.

We can apply this approach to weighted networks. Consider a weighted graph with integer weighted links. Transform each link of the weight $n$ into $n$ parallel links of the weight 1 . We obtain a multigraph. The shortest path between two nodes is determined the same way as in the unweighted graph. But the number of geodesics becomes larger because of the multi-links. If the nodes $i_{1}$ and $i_{2}$ are connected by $m$ links and the nodes $i_{2}$ and $i_{3}$ are connected by $n$ links then the nodes $i_{1}$ and $i_{3}$ are connected by $m \cdot n$ paths. Applying the formula (6) to the nodes of multigraph we derive the centrality value for weighted graph.

\section{Example 1.}

Consider as a simple example the weighted graph (Fig. 1) with the matrix of weights:

\begin{tabular}{|c|c|c|c|}
\hline & $A$ & $B$ & $C$ \\
\hline$A$ & 0 & 1 & 3 \\
\hline$B$ & 1 & 0 & 2 \\
\hline$C$ & 3 & 2 & 0 \\
\hline$D$ & 1 & 0 & 0 \\
\hline
\end{tabular}

First, calculate the betweenness centrality (1) without taking into account the weights. The nodes $B$ and $C$ have the equal values for centrality (see Table 1). In case of weighted graph it is intuitively clear that the node $C$ has a centrality value larger than a value of the node $B$ because $C$ is connected with the node $A$ with larger weight than with the node $B$.

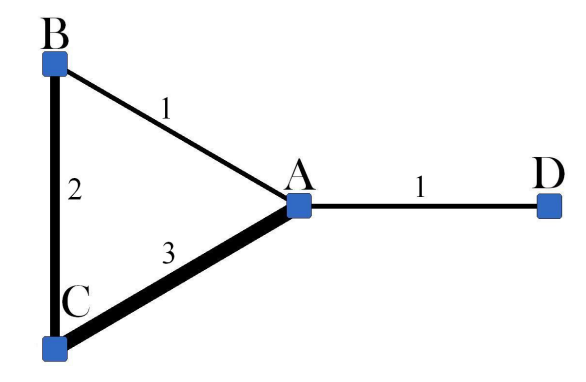

Figure 1. Weighted graph.

Transform this graph into multigraph (Fig. 2) and calculate the Myerson value. We begin from the node $A$. Enumerate all geodesics which contain the node $A$. 


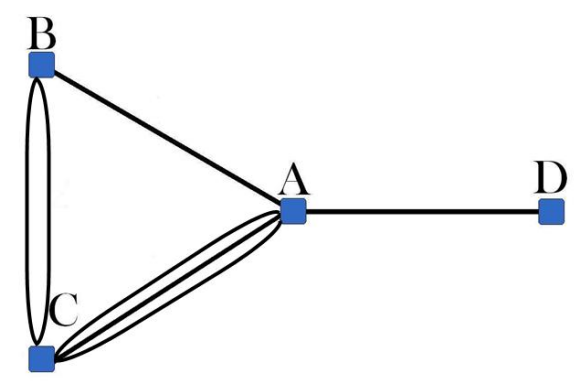

Figure 2. Multigraph.

The paths of length 1: $\{A, B\},\{A, D\}$; and three paths $\{A, C\}$. Thus, $\sigma_{1}(A)=5$.

The paths of length 2: $\{B, A, D\}$, three paths $\{C, A, D\}$ because $A$ and $C$ are connected by three links, so $\sigma_{2}(A)=4$.

Then by formula (6) we have

$$
Y_{A}(G)=\frac{5}{2} r+\frac{4}{3} r^{2}
$$

In a similar manner for the other players we obtain

$$
\begin{gathered}
Y_{B}(G)=\frac{3}{2} r+\frac{1}{3} r^{2} . \\
Y_{C}(G)=\frac{5}{2} r+r^{2} . \\
Y_{D}(G)=\frac{1}{2} r+\frac{4}{3} r^{2} .
\end{gathered}
$$

The results of calculation of the Myerson centrality measure for $r=0.2$ are presented in Table 1 . It confirms our expectation that the centralities of nodes $B$ and $C$ are different in the weighted setting.

The complexity of this algorithm depends on an algorithm of determination of shortest paths in the network. In [15] the algorithm based on the generating function for unweighted graph is proposed. This algorithm can be converted for the weighted graph.

Consider the tree $g_{p}=(N, E)$ with the root in the node $p$. Introduce the generating function

$$
\varphi_{p}(x)=\sum_{k=1}^{L} \alpha_{k}^{p} x^{k}
$$

where $\alpha_{k}^{p}$ is the number of paths which consist of $k$ nodes (length $k-1$ ) and contain the node $p$.

To find this value we use a modified algorithm proposed by Jamison [13] for computing the generating function for the number of sub-trees of a tree $g$ which contain $k$ nodes of the tree $g$. 
Table 1. Myerson centrality for weighted graph from four nodes

\begin{tabular}{|c|c|c|c|c|}
\hline Nodes & $A$ & $B$ & $C$ & $D$ \\
\hline Classical betweenness centrality & 2 & 0 & 0 & 0 \\
\hline $\begin{array}{c}\text { Myerson } \\
\text { centrality }(r=0.2)\end{array}$ & 0.5533 & 0.3133 & 0.5400 & 0.1534 \\
\hline
\end{tabular}

Let us calculate the generating function via recurrence relations. First, we determine the generating functions in the final nodes (leaves) of the tree $g_{p}$. Let $q$ be such a node. Then,

$$
\varphi_{q}(x)=x .
$$

Denote $l$ the number of players in a path of maximal length $\{p, \ldots, q\}$. Consider the nodes $q$ such that the length of path $\{p, \ldots, q\}$ is equal to $l-1$. If it is not the root $p$ then

$$
\varphi_{q}(x)=x\left(1+\sum_{i=1}^{d} \varphi_{q_{i}}(x)\right)
$$

where the sum is calculated in all descendants $q_{i}, i=1, \ldots, d$ of the node $q$. Continue the process until $l=2$.

For $l=2$ the generating function is determined for all descendants of the node $p$. Then, we have

$$
\varphi_{p}(x)=x\left(1+\sum_{i=1}^{d} \varphi_{q_{i}}(x)+\sum_{i \neq j} \varphi_{q_{i}}(x) \varphi_{q_{j}}(x)\right)
$$

where the sum is calculated in all descendants $q_{i}, i=1, \ldots, d$ of the node $p$.

The proof is based on the following arguments. Let $q_{1}, \ldots, q_{d}$ are the descendants of the node $q \neq p$. Then any path from the node $q$ to a node $s$ in the tree $g_{q}$ passes through one of nodes $q_{i}$, and the difference in path lengths is equal to unity.

But if $q=p$ then to paths containing $k$ nodes with the origin in node $p$ we must add paths with $k$ nodes which pass through the node $p$ and can be composed from paths containing $k_{1}<k$ nodes in the tree $g_{q_{i}}$ and paths containing $k-k_{1}$ nodes in the tree $g_{q_{j}}$, where $i \neq j$. The number of such composed paths is determined by the product in the second sum of expression (8).

This method to determine the number of paths using the generating function can be applied for the weighted graph with the only difference that when we rise up to the root, the sub-tree in any node is copied as many times as there are parallel links in the node. That is, in all nodes we calculate $\varphi_{q}(x)=$ $x\left(1+\sum_{i} w\left(q, q_{i}\right) \varphi_{q_{i}}(x)\right)$, where $q_{i}$ are offsprings of $q$ and $w\left(q, q_{i}\right)$ is weight of the link $\left(q, q_{i}\right)$. But in the root of the tree 


$$
\varphi_{p}(x)=x\left(1+\sum_{i=1}^{d} w\left(p, q_{i}\right) \varphi_{q_{i}}(x)+\sum_{i \neq j} w\left(p, q_{i}\right) \varphi_{q_{i}}(x) \cdot w\left(p, q_{j}\right) \varphi_{q_{j}}(x)\right)
$$

Example 2. We demonstrate the method using the generating function to calculate the centrality of the node 4 for the tree presented on Fig. 3. Let node 4 be the root of the tree.

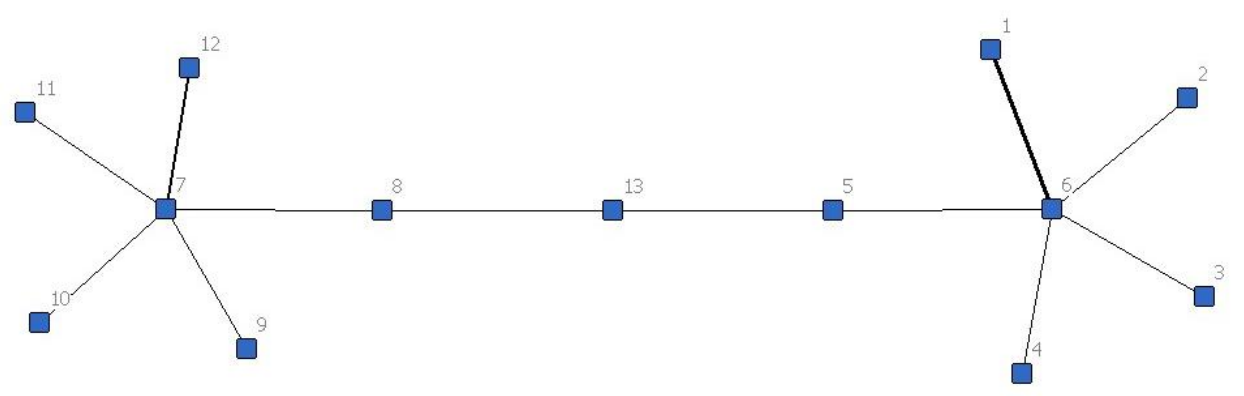

Figure 3. Two weighted stars.

For the final nodes (leaves) of the tree we set

$\varphi_{6}(x)=\varphi_{7}(x)=\varphi_{8}(x)=\varphi_{9}(x)=\varphi_{10}(x)=\varphi_{11}(x)=\varphi_{12}(x)=\varphi_{13}(x)=x$.

For other nodes we calculate

$\varphi_{1}(x)=x\left(1+\varphi_{6}(x)+2 \cdot \varphi_{7}(x)+\varphi_{8}(x)+2 \cdot \varphi_{9}(x)\right)=x(1+6 x)$

$\varphi_{5}(x)=x\left(1+3 \cdot \varphi_{10}(x)+\varphi_{11}(x)+2 \cdot \varphi_{12}(x)+\varphi_{13}(x)\right)=x(1+7 x)$;

$\varphi_{2}(x)=x\left(1+\varphi_{1}(x)\right)=x\left(1+x+6 x^{2}\right)$

$\varphi_{3}(x)=x\left(1+\varphi_{2}(x)\right)=x\left(1+x+x^{2}+6 x^{3}\right) ;$

And for the root 4 by formula (9) we obtain

$\varphi_{4}(x)=x\left(1+4 \cdot \varphi_{3}(x)+\varphi_{5}(x)+4 \cdot \varphi_{3}(x) \cdot \varphi_{5}(x)\right)=x+5 x^{2}+15 x^{3}+36 x^{4}+56 x^{5}+52 x^{6}+168 x^{7}$.

Thus, $\sigma_{1}(4)=\alpha_{2}^{4}=5$;

$\sigma_{2}(4)=\alpha_{3}^{4}=15$;

$\sigma_{3}(4)=\alpha_{4}^{4}=36$

$\sigma_{4}(4)=\alpha_{5}^{4}=56$

$\sigma_{5}(4)=\alpha_{6}^{4}=52$;

$\sigma_{6}(4)=\alpha_{7}^{4}=168$.

By formula (6), we finally obtain

$$
Y_{4}(G)=\frac{5}{2} r+5 r^{2}+9 r^{3}+\frac{56}{5} r^{4}+\frac{26}{3} r^{5}+\frac{168}{7} r^{6} .
$$




\section{Electric measure of centrality based on Kirchhoff's law}

Consider a weighted graph $G=(N, E, W)$, where $N$ is the set of nodes, $E$ is the set of links, and $W$ is the matrix of weights:

$$
W(G)=\left(\begin{array}{cccc}
0 & w_{1,2} & \ldots & w_{1, n} \\
w_{2,1} & 0 & \ldots & w_{2, n} \\
\vdots & \vdots & \ddots & \vdots \\
w_{n, 1} & w_{n, 2} & \ldots & 0
\end{array}\right)
$$

where $w_{i, j} \geq 0$ is the weight of the link connecting the nodes $i$ and $j, n=|N|$ is the number of nodes. Note that $w_{i, j}=0$ if nodes $i$ and $j$ are not adjacent. Let $G$ be the undirected graph, i.e. $w_{i, j}=w_{j, i}$.

Next we introduce the diagonal degree matrix:

$$
D(G)=\left(\begin{array}{cccc}
d_{1} & 0 & \ldots & 0 \\
0 & d_{2} & \ldots & 0 \\
\vdots & \vdots & \ddots & \vdots \\
0 & 0 & \ldots & d_{n}
\end{array}\right)
$$

where $d_{i}=\sum_{j=1}^{n} w_{i, j}$ is the sum of weights of the edges which are adjacent to node $i$ in graph $G$.

Definition. The Laplacian matrix $L(G)$ for weighted graph $G$ is defined as

$$
L(G)=D(G)-W(G)=\left(\begin{array}{cccc}
d_{1} & -w_{1,2} & \ldots & -w_{1, n} \\
-w_{2,1} & d_{2} & \ldots & -w_{2, n} \\
\vdots & \vdots & \ddots & \vdots \\
-w_{n, 1} & -w_{n, 2} & \ldots & d_{n}
\end{array}\right)
$$

Let the graph $G^{\prime}$ be converted from the graph $G$ by extension of an additional node $n+1$ connected with all nodes of the graph $G$ with the links of constant conductivity $\delta$. Thus, we obtain the Laplacian matrix for the modified graph $G^{\prime}$ as:

$$
L\left(G^{\prime}\right)=D\left(G^{\prime}\right)-W\left(G^{\prime}\right)=\left(\begin{array}{ccccc}
d_{1}+\delta & -w_{1,2} & \ldots & -w_{1, n} & -\delta \\
-w_{2,1} & d_{2}+\delta & \ldots & -w_{2, n} & -\delta \\
\vdots & \vdots & \ddots & \vdots & \vdots \\
-w_{n, 1} & -w_{n, 2} & \ldots & d_{n}+\delta & -\delta \\
-\delta & -\delta & \ldots & -\delta & \delta n
\end{array}\right)
$$

Suppose that a unit of current enters into the node $s \in V$ and the node $n+1$ is grounded. Let $\varphi_{i}^{s}$ be the electric potential at node $i$ when an electric charge is located at the node $s$. The vector of all potentials $\varphi^{s}\left(G^{\prime}\right)=\left[\varphi_{1}^{s}, \ldots, \varphi_{n}^{s}, \varphi_{n+1}^{s}\right]^{T}$ for the nodes of the graph $G^{\prime}$ is determined by the following system of equations (the Kirchhoff's law):

$$
\varphi^{s}\left(G^{\prime}\right)=L\left(G^{\prime}\right)^{-1} b_{s}^{\prime}
$$


10Avrachenkov K.E., Mazalov V.V., Trukhina L.I., Tsinguev B.T. /Game-Theoretic Centrality Measures for Weighted Graphs

where $b_{s}^{\prime}$ is the vector of $n+1$ components with the values:

$$
b_{s}^{\prime}(i)= \begin{cases}1 & i=s, \\ 0 & \text { otherwise }\end{cases}
$$

Laplacian matrix (10) is singular. Without loss of generality, we can assume that the potential in the node $n+1$ is equal to 0 . Thus, from (11) it follows:

$$
\tilde{\varphi}^{s}\left(G^{\prime}\right)=\tilde{L}\left(G^{\prime}\right)^{-1} b_{s}
$$

where $\tilde{\varphi}^{s}\left(G^{\prime}\right), \tilde{L}\left(G^{\prime}\right)$ and $b_{s}$ are obtained from (11) by deleting of row and column corresponding to the node $n+1$. Notice that in $\varphi^{s}\left(G^{\prime}\right)$ and $b_{s}^{\prime}$ zero elements are deleted. It yields

$$
\tilde{\varphi}^{s}\left(G^{\prime}\right)=[D(G)-W(G)+\delta I]^{-1} b_{s},
$$

where $I$ is a unity matrix of size $n$.

The potential values can be determined up to a constant. Then, we can consider the vector $\tilde{\varphi}^{s}\left(G^{\prime}\right)$ as the potential values for the nodes of the graph $G$, that is,

$$
\tilde{\varphi}^{s}(G)=[L(G)+\delta I]^{-1} b_{s} .
$$

Rewrite (15) in the following form:

$$
\begin{gathered}
\tilde{\varphi}^{s}(G)=[(D(G)+\delta I)-W(G)]^{-1} b_{s}= \\
=\left[I-(D(G)+\delta I)^{-1} D(G) D^{-1}(G) W(G)\right]^{-1}(D(G)+\delta I)^{-1} b_{s} .
\end{gathered}
$$

The matrices $(D(G)+\delta I)^{-1}$ and $(D(G)+\delta I)^{-1} D(G)$ are diagonal with the elements $\frac{1}{d_{i}+\delta}$ and $\frac{d_{i}}{d_{i}+\delta}, i=$ $1, \ldots, n$, denote it as $D_{1}$ and $D_{2}$, respectively. The matrix $D^{-1}(G) W(G)$ is stochastic. Denote it as $P$. Consequently,

$$
\tilde{\varphi}^{s}(G)=\left[I-D_{2} P\right]^{-1} D_{1} b_{s}=\sum_{k=0}^{\infty}\left(D_{2} P\right)^{k} D_{1} b_{s} .
$$

From (16) it follows that the potential vector can be calculated by the recursion:

$$
\tilde{\varphi}_{k+1}^{s}(G)=D_{2} P \tilde{\varphi}_{k}^{s}(G)+D_{1} b_{s}, \quad \tilde{\varphi}_{0}^{s}(G)=0 .
$$

According to Ohms law, the current let-through the link $e=(i, j)$ is $x_{e}^{s}=\left|\varphi_{i}^{s}-\varphi_{j}^{s}\right| \cdot w_{i, j}$. Consequently, given that the electric charge is in the node $s$, the mean value of the current passing through node $i$ is as follows:

$$
x^{s}(i)=\frac{1}{2}\left(b_{s}(i)+\sum_{e: i \in e} x_{e}^{s}\right),
$$

where

$$
b_{s}(i)= \begin{cases}1 & i=s \\ 0 & \text { otherwise. }\end{cases}
$$

Thus, the electric centrality measure in node $i$ for the weighted graph, $C F_{B}(i)$, can be determined by the formula:

$$
C F_{B}(i)=\frac{1}{n} \sum_{s \in V} x^{s}(i) \text {. }
$$




\section{The Myerson value and cooperative game corresponding to the model of electric circuit}

The model of electric circuit related to the weighted graph can be used for the determination of the characteristic function of a cooperative game. The utility of a coalition in this game is a total current passing through the nodes of the corresponding coalition. For that let us slightly modify the model eliminating the charge. Notice that the component $\frac{1}{2} b_{s}(i)$ in formula (17) gives the equal impact for all nodes of the coalition. So, this part of the current is not important for the comparing the nodes in the coalition.

Thus, determine the characteristic function in the cooperative game as

$$
v(K)=\frac{1}{|K|} \sum_{s \in K} x_{K}^{s}, \quad K \subset V,
$$

where

$$
x_{K}^{s}=\frac{1}{2} \sum_{i \in K} \sum_{e: i \in e} x_{i}^{s} .
$$

As a measure of centrality we can take the Myerson value in the cooperative game with the above characteristic function.

Next we present the results of computer simulations for all introduced measures of the network centrality.

\section{Example 3.}

Let us find the electric centrality for the weighted graph from Example 1. The results of the calculations are presented in Table 2. The data of Table 2 confirm once more that the node $C$ has a value of centrality larger than the centrality of the node $B$.

Table 2. Measures of centrality for weighted graph with four nodes

\begin{tabular}{|c|c|c|c|c|}
\hline Nodes & $A$ & $B$ & $C$ & $D$ \\
\hline Classical betweenness centrality & 2 & 0 & 0 & 0 \\
\hline $\begin{array}{c}\text { Electric } \\
\text { centrality }(\delta=1)\end{array}$ & 0.4018 & 0.2679 & 0.3348 & 0.2277 \\
\hline $\begin{array}{c}\text { Electric centrality } \\
\text { by Myerson }(\delta=0.5)\end{array}$ & 0.3423 & 0.1862 & 0.2196 & 0.1204 \\
\hline
\end{tabular}

\section{Example 4.}

Consider the graph of two stars presented in Fig. 3. First, let us calculate the electric centrality and the Myerson centrality for the unweighted network and compare it with the PageRank Centrality. The results of calculations are presented in Table 3. 
Table 3. Unweighted graph. The electric centrality $(\delta=1)$, the Myerson vector $(r=0.2)$ and PageRank Centrality $(\alpha=0.85)$

\begin{tabular}{|c|c||c|c||c|c|}
\hline Nodes & $\begin{array}{c}\text { Centrality } \\
\left(C F_{B}\right)\end{array}$ & Nodes & $\begin{array}{c}\text { Centrality } \\
\text { by Myerson }\end{array}$ & Nodes & PageRank \\
\hline 1 & 0.2105 & 1 & 0.6588 & 1 & 0.1998 \\
5 & 0.2105 & 5 & 0.6588 & 5 & 0.1998 \\
2 & 0.1131 & 2 & 0.2955 & 2 & 0.0789 \\
4 & 0.1131 & 4 & 0.2955 & 4 & 0.0789 \\
3 & 0.1089 & 3 & 0.2635 & 3 & 0.0786 \\
6 & 0.0716 & 6 & 0.1557 & 6 & 0.0455 \\
7 & 0.0716 & 7 & 0.1557 & 7 & 0.0455 \\
8 & 0.0716 & 8 & 0.1557 & 8 & 0.0455 \\
9 & 0.0716 & 9 & 0.1557 & 9 & 0.0455 \\
10 & 0.0716 & 10 & 0.1557 & 10 & 0.0455 \\
11 & 0.0716 & 11 & 0.1557 & 11 & 0.0455 \\
12 & 0.0716 & 12 & 0.1557 & 12 & 0.0455 \\
13 & 0.0716 & 13 & 0.1557 & 13 & 0.0455 \\
\hline
\end{tabular}


We see that all three methods evaluate the nodes at the same order. The coefficient of correlation between the values of electric centrality and Myerson centrality is equal to 0.999; the coefficient of correlation between the values of electric centrality and PageRank Centrality is equal to 0.996 .

Now consider this network of two stars with the weights and calculate the centrality measures for this case; the results are presented in Table 4.

Table 4. Weighted graph. The electric centrality $(\delta=1)$; the Myerson vector $(r=0.2)$

\begin{tabular}{|c|c||c|c|}
\hline Nodes & $\begin{array}{c}\text { Centrality } \\
\left(C F_{B}\right)\end{array}$ & Nodes & $\begin{array}{c}\text { Centrality } \\
\text { by Myerson }\end{array}$ \\
\hline 5 & 0.2399 & 5 & 1.2519 \\
1 & 0.2316 & 1 & 0.9999 \\
4 & 0.1363 & 4 & 0.7942 \\
3 & 0.1307 & 3 & 0.7262 \\
2 & 0.1173 & 10 & 0.5291 \\
10 & 0.0856 & 2 & 0.3969 \\
12 & 0.0814 & 12 & 0.3794 \\
7 & 0.0811 & 7 & 0.3408 \\
9 & 0.0811 & 9 & 0.3408 \\
11 & 0.0722 & 11 & 0.2030 \\
13 & 0.0722 & 13 & 0.2030 \\
6 & 0.0722 & 6 & 0.1837 \\
8 & 0.0721 & 8 & 0.1837 \\
\hline
\end{tabular}

\section{The results of computer simulations for social network}

Here we consider the weighted graph derived from the popular Russian social network VKontakte. The graph corresponds to the community devoted to game theory. This community consists of 483 participants. As a weight of the link we take the number of common friends between the participants. In fact, the probability that two participants are familiar depends on the number of common friends. This approach is often used in the social network analysis.

In Fig. 4 the principal component of the community "Game Theory" consists of 275 nodes. It is difficult to see from Fig. 4 which nodes are more important for the connection in the community. We converted this graph to a new graph by deleting the links whose weights are less than three. This new 


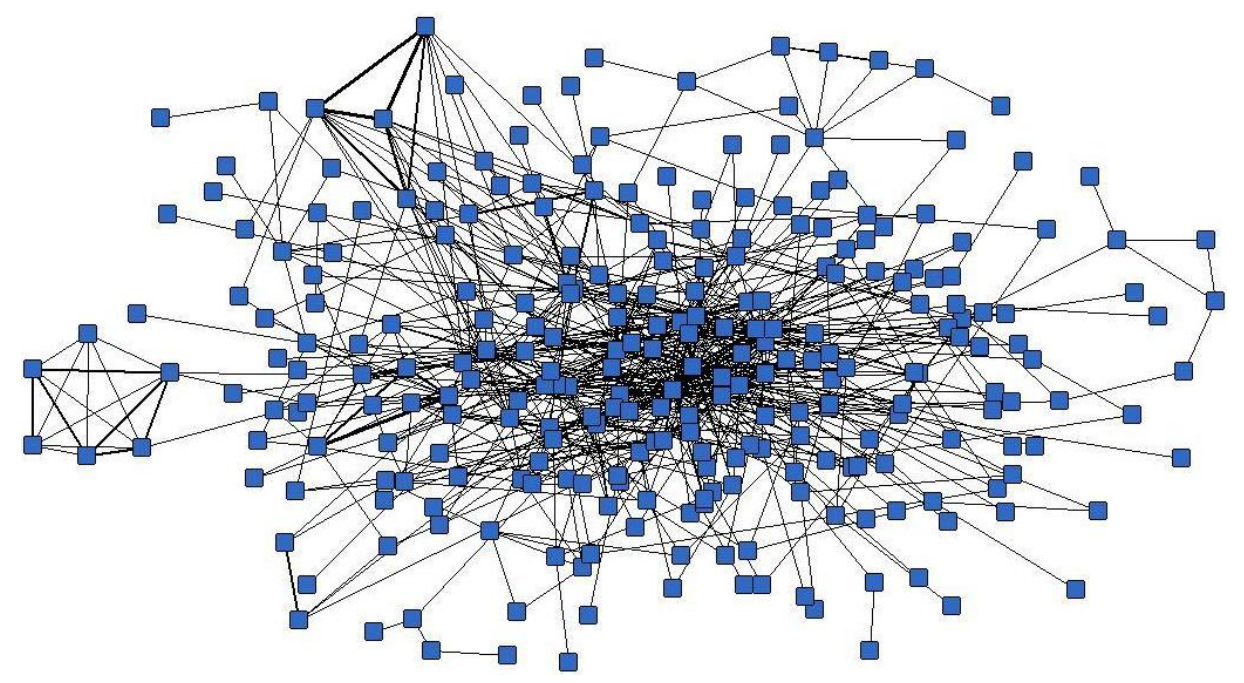

Figure 4. Principal component of the community Game Theory in the social network VKontakte (275 nodes).

weighted graph is presented in Fig. 5. The thickness of a link depends on the weight of the link, i.e. on the number of common friends.

The results of computing the electric centrality for the social network VKontakte are given in Table 5. Here the parameter $\delta=1$. In Table 6 there are results of computations for the Myerson centrality for the network with $r=0.2$. It is useful to compare these values of centrality with the results corresponding to the classical notation of centrality using the shortest paths [18] for the parameter $\alpha=1.5$.

From Tables 5-7 we find that all three methods ranked two main nodes 1 and 8 in the same order. The nodes 52, 56, 63, 69 are evaluated also as having large centrality. We can compare these methods by computing the correlation between the rankings. The coefficient of correlation between the values of electric centrality and Myerson centrality is equal to 0.929; the coefficient of correlation between the values of electric centrality and the centrality given by the program "tnet" is equal to 0.953 ; the coefficient of correlation between the Myerson centrality and the centrality given by the program "tnet" is equal to 0.948 . Thus, our main conclusion that the electric centrality measure correlates well with the game-theoretic based centrality measures and less well with PageRank.

The figures of weighted graphs are prepared using the component NETDRAW from the program package "UCINET" [3]. 


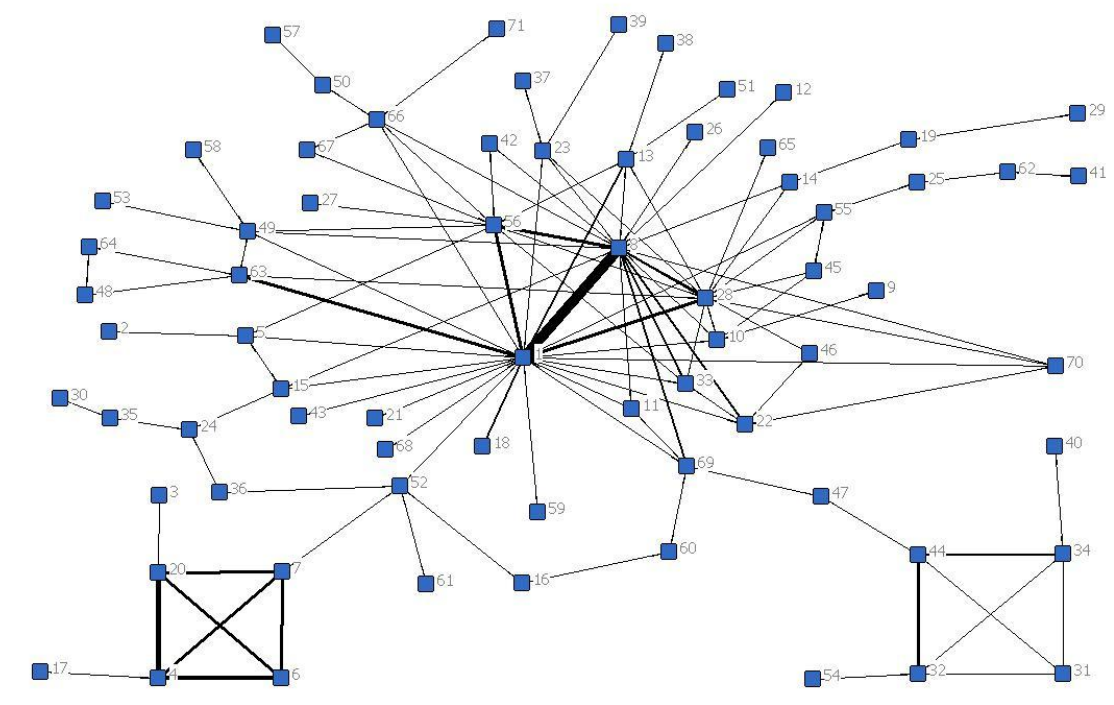

Figure 5. Principal component of the community Game Theory in the social network VKontakte after deleting the links weighted less than 3 (71 nodes). 
Table 5. Social network VKontakte. The electric centrality $(\delta=1))$

\begin{tabular}{|c|c|c|c|c|c|c|c|}
\hline Nodes & $\begin{array}{c}\text { Centrality } \\
\left(C F_{B}\right)\end{array}$ & Nodes & $\begin{array}{c}\text { Centrality } \\
\left(C F_{B}\right)\end{array}$ & Nodes & $\begin{array}{c}\text { Centrality } \\
\left(C F_{B}\right)\end{array}$ & Nodes & $\begin{array}{c}\text { Centrality } \\
\left(C F_{B}\right)\end{array}$ \\
\hline 1 & 0.3267 & 14 & 0.04321 & 11 & 0.0247 & 71 & 0.0181 \\
\hline 8 & 0.2521 & 22 & 0.04151 & 48 & 0.0241 & 38 & 0.0179 \\
\hline 56 & 0.1095 & 5 & 0.0415 & 67 & 0.0236 & 61 & 0.0178 \\
\hline 28 & 0.1045 & 4 & 0.0412 & 70 & 0.0222 & 57 & 0.0178 \\
\hline 52 & 0.0915 & 34 & 0.0395 & 64 & 0.0213 & 21 & 0.0174 \\
\hline 69 & 0.0872 & 20 & 0.03908 & 18 & 0.0203 & 43 & 0.0174 \\
\hline 63 & 0.0778 & 25 & 0.03870 & 27 & 0.0197 & 59 & 0.0174 \\
\hline 66 & 0.0738 & 32 & 0.0387 & 12 & 0.0197 & 26 & 0.0174 \\
\hline 7 & 0.0640 & 60 & 0.03629 & 31 & 0.0196 & 65 & 0.0174 \\
\hline 15 & 0.0638 & 50 & 0.03553 & 46 & 0.0193 & 51 & 0.0172 \\
\hline 49 & 0.0632 & 36 & 0.03498 & 37 & 0.0191 & 30 & 0.0172 \\
\hline 23 & 0.0615 & 45 & 0.0328 & 42 & 0.0189 & 40 & 0.0170 \\
\hline 13 & 0.0602 & 35 & 0.0324 & 39 & 0.0189 & 41 & 0.0169 \\
\hline 24 & 0.0590 & 16 & 0.0317 & 58 & 0.0188 & 17 & 0.0166 \\
\hline 55 & 0.0553 & 62 & 0.0314 & 68 & 0.0185 & 3 & 0.0166 \\
\hline 44 & 0.0530 & 19 & 0.0292 & 2 & 0.0183 & 54 & 0.0165 \\
\hline 10 & 0.0516 & 33 & 0.0291 & 9 & 0.0182 & 29 & 0.0156 \\
\hline 47 & 0.0470 & 6 & 0.0277 & 53 & 0.0182 & & \\
\hline
\end{tabular}


Table 6. Social network VKontakte. The Myerson centrality $(r=0.2)$

\begin{tabular}{|c|c||c|c||c|c||c|c|}
\hline Nodes & Centrality & Nodes & Centrality & Nodes & Centrality & Nodes & Centrality \\
\hline 1 & 4806,07 & 6 & 366,08 & 17 & 186,38 & 57 & 92,44 \\
8 & 3732,69 & 64 & 350,86 & 5 & 185,13 & 38 & 92,29 \\
52 & 1544,06 & 15 & 344,85 & 54 & 182,45 & 9 & 85,35 \\
63 & 1436,29 & 55 & 339,23 & 49 & 165,20 & 41 & 85,00 \\
7 & 1365,36 & 67 & 326,77 & 35 & 156,80 & 29 & 73,66 \\
56 & 1328,20 & 13 & 306,28 & 3 & 150,95 & 70 & 71,23 \\
69 & 1253,62 & 42 & 304,48 & 33 & 148,27 & 51 & 69,26 \\
47 & 972,53 & 23 & 302,27 & 62 & 146,91 & 58 & 65,99 \\
12 & 971,42 & 34 & 285,07 & 50 & 142,70 & 21 & 65,73 \\
44 & 904,69 & 46 & 278,55 & 60 & 131,68 & 43 & 65,73 \\
27 & 612,69 & 66 & 274,13 & 37 & 129,22 & 59 & 65,73 \\
28 & 587,49 & 26 & 265,25 & 40 & 116,66 & 16 & 65,64 \\
4 & 554,02 & 22 & 250,80 & 11 & 109,72 & 36 & 60,59 \\
18 & 540,42 & 24 & 240,70 & 68 & 109,42 & 61 & 59,01 \\
32 & 533,48 & 10 & 231,91 & 39 & 107,95 & 71 & 50,82 \\
48 & 526,10 & 19 & 217,01 & 30 & 102,57 & 31 & 45,97 \\
14 & 505,58 & 45 & 210,66 & 65 & 102,37 & 53 & 41,45 \\
20 & 448,98 & 25 & 199,09 & 2 & 96,99 & & \\
\hline & & & & & & & \\
\hline
\end{tabular}


Table 7. Social network VKontakte. The results of the program package tnet $(\alpha=1.5))$

\begin{tabular}{|c|c||c|c||c|c||c|c|}
\hline Nodes & Centrality & Nodes & Centrality & Nodes & Centrality & Nodes & Centrality \\
\hline 1 & 1846 & 14 & 136 & 9 & 0 & 43 & 0 \\
8 & 1398 & 25 & 136 & 11 & 0 & 45 & 0 \\
52 & 500 & 4 & 69 & 12 & 0 & 46 & 0 \\
69 & 494 & 5 & 69 & 17 & 0 & 48 & 0 \\
47 & 384 & 10 & 69 & 18 & 0 & 51 & 0 \\
44 & 331 & 19 & 69 & 21 & 0 & 53 & 0 \\
63 & 331 & 20 & 69 & 26 & 0 & 54 & 0 \\
7 & 325 & 28 & 69 & 27 & 0 & 57 & 0 \\
55 & 265 & 32 & 69 & 29 & 0 & 58 & 0 \\
15 & 228 & 35 & 69 & 30 & 0 & 59 & 0 \\
66 & 203 & 50 & 69 & 31 & 0 & 61 & 0 \\
24 & 194 & 62 & 69 & 33 & 0 & 64 & 0 \\
13 & 137 & 36 & 27 & 37 & 0 & 65 & 0 \\
22 & 137 & 16 & 8 & 38 & 0 & 67 & 0 \\
23 & 137 & 60 & 8 & 39 & 0 & 68 & 0 \\
34 & 137 & 2 & 0 & 40 & 0 & 70 & 0 \\
49 & 137 & 3 & 0 & 41 & 0 & 71 & 0 \\
56 & 137 & 6 & 0 & 42 & 0 & & \\
\hline
\end{tabular}


Avrachenkov K.E., Mazalov V.V., Trukhina L.I., Tsinguev B.T./Game-Theoretic Centrality Measures for Weighted Graphs19

\section{References}

[1] Aumann, R., Myerson, R.: Endogenous formation of links between players and coalitions: an application of the Shapley value, in: The Shapley value, Cambridge University Press, 1988, 175-191.

[2] Avrachenkov, K., Litvak, N., Medyanikov, V. and Sokol, M.: Alpha current flow betweenness centrality, in: Proceedings of WAW 2013, (also LNCS v.8305), 2013, 106-117.

[3] Borgatti, S.P., Everett, M.G. and Freeman, L.C.: Ucinet for Windows: Software for Social Network Analysis, Harvard, MA: Analytic Technologies, 2002.

[4] Borm, P., Owen, G., Tijs, S.: On the position value for communication situations, SIAM J. on Discrete Math., 5(3), 1992, 305-320.

[5] Borm, P., van den Nouweland, A., Tijs, S.: Cooperation and communication restrictions: a survey, in: Imperfections and Behavior in Economic Organizations, Kluwer Acad. Publ., Boston, 1994.

[6] Brandes, U.: A faster algorithm for betweenness centrality, Journal of Mathematical Sociology, 25, 2001, 163-177.

[7] Brandes, U., Fleischer, D.: Centrality measures based on current flow, in: Proceedings of the 22nd annual conference on Theoretical Aspects of Computer Science, 2005, 533-544.

[8] Calvo, E., Lasaga, J., van den Nouweland, A.: Values of games with probabilistic graphs, Math. Social Sci., 37, 1999, 79-95.

[9] Freeman, L.C.: A set of measures of centrality based on betweenness, Sociometry, 40, 1977, 35-41.

[10] Jackson, M.O.: Allocation rules for network games, Games and Econ. Behav., 51(1), 2005, $128-154$.

[11] Jackson, M.O., Wolinsky, J.: A strategic model of social and economic networks, J. Econ. Theory, 71(1), 1996, 44-74.

[12] Jackson, M.O.: Social and economic networks, Princeton University Press, 2008.

[13] Jamison, R.E.: Alternating Whitney sums and matchings in trees, part 1. Discrete Math., 67, 1987, 177-189.

[14] Mazalov, V.: Mathematical Game Theory and Applications, Wiley, 2014.

[15] Mazalov, V.V., Trukhina, L.I.: Generating functions and the Myerson vector in communication networks, Discrete Mathematics and Applications 24(5), 2014, 295-303.

[16] Myerson, R.B.: Graphs and cooperation in games, Math. Oper. Res., 2, 1977, 225-229.

[17] Newman, M.E.J.: A measure of betweenness centrality based on random walks, Social networks, 27, 2005, 39-54.

[18] Opsahl, T., Agneessens, F., Skvoretz, J.: Node centrality in weighted networks: generalizing degree and shortest paths, Social Networks, 32, 2010, 245-251.

[19] Slikker, M.: Link monotonic allocation schemes, Int. Game Theory Review, 7(4), 2005, 473-489.

[20] Slikker, M., Gilles, R.P., Norde, H., Tijs, S.: Directed networks, allocation properties and hierarchy formation, Math. Social Sci. 49(1), 2005, 55-80.

[21] Talman, D., Yamamoto, Y.: Average tree solutions and subcore for acyclic graph games, J. Oper. Res. Soc. Japan, 51(3), 2008, 187-201. 